\title{
Selenium Deficiency Causes Inflammatory Injury in the Bursa of Fabricius of Broiler Chickens by Activating the Toll-like Receptor Signaling Pathway
}

\author{
Yu Bai ${ }^{1,2,3} \cdot$ Ruili Zhang ${ }^{1,2}$. Qing Liu ${ }^{1,2} \cdot$ Rong Guo ${ }^{1}$. Guangxing $\mathrm{Li}^{1} \cdot$ Bin Sun ${ }^{4} \cdot$ Di Zhang $^{1} \cdot$ Yang Chen ${ }^{1}$. \\ Xiaodan Huang ${ }^{1,2}$ (1)
}

Received: 17 February 2021 / Accepted: 21 March 2021 / Published online: 25 March 2021

C The Author(s), under exclusive licence to Springer Science+Business Media, LLC, part of Springer Nature 2021

\begin{abstract}
The aim of our study was to observe the effect of selenium (Se) deficiency on inflammatory injury in the bursa of Fabricius of broiler chickens and to determine the role of the Toll-like receptor (TLR)/myeloid differential protein-88 (MyD88)/nuclear factor- $\mathrm{KB}(\mathrm{NF}-\mathrm{kB})$ signaling pathway during this process. Here, we revealed that severe inflammatory injury occurred in the broiler bursa of Fabricius with Se deficiency via histopathology. Moreover, the ultrastructural pathological results showed that the nuclear, mitochondrial, endoplasmic reticulum and cytomembrane structures were damaged to varying degrees. Additionally, interleukin-2 (IL-2), interleukin-6 (IL-6), and interferon (IFN- $\gamma)$ mRNA expression was markedly upregulated in the broiler bursa of Fabricius with Se deficiency. Furthermore, TLR, toll-interleukin-1 receptor domain-containing adapter-inducing interferon- $\beta$ (TRIF), $M y D 88$, and $N F-\kappa B$ mRNA expression was also markedly elevated in the broiler bursa of Fabricius with Se deficiency. The above results suggested that Se deficiency increases the expression of numerous proinflammatory cytokines and is probably due to the activation of the TLR/MyD88/NF-KB signaling pathway, which causes inflammatory injury in the bursa of Fabricius of broiler chickens. Our findings provide a theoretical reference for further studying the underlying mechanism of Se deficiency-induced inflammatory injury in the bursa of Fabricius of broiler chickens.
\end{abstract}

Keywords Selenium deficiency $\cdot$ Inflammatory injury $\cdot$ Bursa of Fabricius $\cdot$ Toll-like receptor

\section{Introduction}

Selenium (Se) is an essential trace element for physiological activities in the body $[1,2]$. Organic Se sources rather than inorganic sources are increasingly advocated as Se supplements in livestock and poultry farming [3]. Earlier studies demonstrated that Se plays a vital role in the growth and metabolism of animals

Yu Bai, Ruili Zhang and Qing Liu contributed equally to this work.

Xiaodan Huang

hxd790125@neau.edu.cn

1 Department of Veterinary Pathology, College of Veterinary Medicine, Northeast Agricultural University, Harbin 150030, China

2 Heilongjiang Key Laboratory for Laboratory Animals and Comparative Medicine, Northeast Agricultural University, Harbin 150030, China

3 Department of Veterinary Pathophysiology, College of Animal Medicine, China Agricultural University, Beijing 100193, China

4 College of Animal Science and Veterinary Medicine, Heilongiiang Bayi Agricultural University, Daqing 163000, China
[4]. For example, Se and vitamin E deficiency causes white muscle disease and cardiomyopathy in animals [4]. Recently, an increasing number of studies have revealed that $\mathrm{Se}$, selenoprotein, and other Se-containing compounds exert antioxidative functions and inhibit apoptosis and autophagy in multiple organs $[1,5,6]$. Se-containing compounds could also mediate anti-inflammatory functions to maintain the balance between inflammatory and anti-inflammatory responses in organs and tissues [7-9]. Se deficiency could result in severe inflammatory injury in organs. Wu et al. found that Se deficiency causes inflammatory injury of the gastrointestinal tract in broilers [10]. Bi et al. found that Se deficiency facilitates inflammation following S. aureus infection in the mouse mammary gland [7]. It is widely believed that $\mathrm{Se}$ is the trace element most closely associated with influenza virus infection [11]. A report showed that early nutritional interventions with selenium, zinc, and vitamin D present the function of raising antiviral resistance against coronavirus disease 2019 (COVID-19) [12]. Therefore, it is necessary to investigate the effect of Se deficiency on organ damage and rational supplementation with Se to improve growth performance and disease resistance in animals. 
Immunity, protects the body from a large number of pathogen attacks and is divided into innate immunity and adaptive immunity based on immunological characteristics. Innate immunity is the first barrier to prevent pathogen invasions into the body. For example, the interferon (IFN) signaling pathway exerts broad antiviral functions and is a critical defense system against viruses during the early stage of infection in animals [13]. The body activates the innate immune response to counter pathogenic factors recognized by pattern recognition receptors (PRRs). Tolllike receptors (TLRs) are one of the most important PRRs in humans and animals. Scientists have successively identified more than 20 kinds of TLRs, and different kinds of TLRs can recognize different pathogenic invaders as foreign. For example, TLR-1 recognizes lipoproteins of the mycobacterium, TLR-4 recognizes lipopolysaccharides (LPS), and TLR-3 and TLR-7 recognize double-stranded RNA and single-stranded RNA from viruses, respectively [14]. Although the immune response protects the body from viruses, bacteria and other pathogens, excessive or persistent immune responses themselves could cause severe inflammatory injury in organs and further impair organ biological functions. For example, influenza virus infection causes a "cytokine storm" (one of the excessive immune response manifestations) to result in acute lung injury, which contributes to high morbidity and mortality worldwide [15]. Recently, emerging evidence has indicated that Se deficiency can directly or indirectly trigger innate immunity in animals.

The bursa of Fabricius, where B lymphocytes mature, is a vital immune organ in broilers. Thus, the healthy growth and metabolism of the bursa of Fabricius is essential for strengthening antiviral or antibacterial abilities and even for the immune effect of vaccines. Numerous studies have revealed that Se plays a fundamentally important role in regulating the immune function of macrophages, B cells, T cells, natural killer cells, and other immune cells $[1,16]$. However, there is little research about the effect of Se deficiency on injury to the bursa of Fabricius and its underlying mechanism during the pathogenic process.

In the present study, we uncovered that Se deficiency markedly elevates enormous pro-inflammatory cytokine mRNA expression, probably dominated by the activation of the TLR/MyD88/NF-kB signaling pathway, thereby causing severe inflammatory injury and multiple organelle damage in the bursa of Fabricius of broilers. Our findings provide a theoretical reference for further studying the underlying mechanism of Se deficiency-induced inflammatory injury in the bursa of Fabricius of broilers.

\section{Materials and methods}

\section{Animals and low-Se diets}

One-day-old healthy broilers (clean grade) were purchased from Yinong Poultry Co., Ltd. (Harbin, China). The feeding

environment was disinfected during the experiment. The brooding temperature was ensured. All of the broilers ate and drank freely. Research staff received training on animal care and handling. The procedures in this study were approved by the Institutional Animal Care and Use Committee of Northeast Agricultural University.

In the present study, the composition of the difference between the low-Se diet and the control diet (complete formula feed) was only the addition of sodium selenite. The Se content of the low-Se diet was $0.004 \mathrm{mg} / \mathrm{kg}$, while the Se content of the control diet was $0.3 \mathrm{mg} / \mathrm{kg}$. The composition of the low-Se diet used in this experiment is shown in Table $1[17,18]$.

\section{Animal experiments}

\section{Experiment 1}

We first observed whether Se deficiency causes inflammatory injury in the broiler bursa of Fabricius. Twelve one-day-old healthy broilers were randomly and averagely divided into a control group (C group) and a low-Se group (L group). Broilers of the $\mathrm{C}$ group were fed complete formula feed, and broilers of the L group were fed the low-Se diet. According to our previous studies, we found that Se deficiency could cause severe inflammatory injury in the broiler spleen and muscular stomach on day 55. Thus, we also collected samples to observe inflammatory injury on day $55[17,18]$. Six broilers per group were anesthetized and sacrificed for pathologic autopsy. Three broiler bursae of Fabricius in each group were fixed in $4 \%$ paraformaldehyde for histopathology, and the remaining 3

Table 1 The composition of the lowSe diet

\begin{tabular}{ll}
\hline Composition & Contents (\%) \\
\hline Corn $^{\text {a }}$ & 63.5 \\
Soybean meal $^{\text {a }}$ & 28.0 \\
Wheat meal $^{\text {a }}$ & 2.84 \\
$\mathrm{NaCl}$ & 0.27 \\
Soybean oil & 1 \\
Choline $_{\mathrm{CaCO}_{3}}$ & 0.07 \\
$\mathrm{CaHPO}_{4}$ & 1.2 \\
DL-methionine & 2.3 \\
Lysine & 0.18 \\
Vitamins & 0.4 \\
Minerals & 0.04 \\
Total & 0.2 \\
\hline
\end{tabular}

Note: ${ }^{a}$ Corn, soybean meal and wheat meal were purchased from Longjiang County, a typical Se-deficient area in Heilongjiang Province, China 
broiler bursa of Fabricius in each group were fixed in 2.5\% glutaraldehyde solution for ultrastructural pathology.

\section{Experiment 2}

Then, we determined whether Se deficiency could elevate proinflammatory cytokine mRNA expression and initiate TLR/MyD88/NF-KB signaling in the bursa of Fabricius. Thirty, one-day-old healthy broilers were randomly and averagely divided into the $\mathrm{C}$ group and the $\mathrm{L}$ group. The treatments of the $\mathrm{C}$ and $\mathrm{L}$ groups were the same as those in experiment 1 . On days 15,35 . and 55,5 broilers per group at every point in time were anesthetized and sacrificed for pathologic autopsy. The bursae of Fabricius were collected and stored at $-80^{\circ} \mathrm{C}$ for real-time quantitative PCR (RT-qPCR).

\section{Histopathology}

The fixed bursae of Fabricius from the $\mathrm{C}$ and $\mathrm{L}$ groups were embedded in paraffin and sectioned for histopathological experiments. Sections were stained with hematoxylin and eosin (H\&E) for microscopy. The results were confirmed and described by an experienced and qualified pathologist.

\section{Transmission electron microscopy test}

The fixed bursae of Fabricius from the $\mathrm{C}$ and $\mathrm{L}$ groups were dehydrated, saturated, embedded, and sectioned for ultrastructural pathology experiments. Sections were stained by uranium acetate and lead citrate staining. The results were described by experienced and qualified research staff.

\section{RT-qPCR}

Total RNA in the bursa of Fabricius was extracted by TRIzol reagent (TransGen, ET101-01, China). Next, cDNA was synthesized using the Prime Script RT Reagent Kit with gDNA Eraser (TaKaRa, RR037A, Japan) and detected by RT-qPCR with Tli RNase H Plus (TaKaRa, SYBR Premix Ex Taq II, Japan). $\beta$-actin gene was used as the loading control gene. The relative mRNA expression was calculated by the $2^{-\triangle \Delta C T}$ method. Primer sequences are listed in Table 2.

\section{Data analysis}

GraphPad Prism 6.0 was used to perform statistical analysis. Data are presented as the mean $\pm \mathrm{SD}$. The statistical significance between two groups was analyzed using one-way ANOVA followed by Student's $t$ test. A value of $P<0.05$ was considered significant.
Table 2 Primer sequences for RT-qPCR

\begin{tabular}{|c|c|}
\hline Gene name & Sequence $\left(5^{\prime}-3^{\prime}\right)$ \\
\hline \multirow[t]{2}{*}{$\beta$-actin } & F: CGTGACTGACTGACTACCT \\
\hline & R: TGATCGAAATCAGTGCGAC \\
\hline \multirow[t]{2}{*}{$I F N-\gamma$} & F: GCTGGGTGGACCTATTATTGTAGAG \\
\hline & R: TTCTTCACGCCATGGAAGGTTG \\
\hline \multirow[t]{2}{*}{$I L-2$} & F: TTCTGCACCACTTCCTTTGAGTC \\
\hline & R: GCGTGCTACTGACCTGAGTATG \\
\hline \multirow[t]{2}{*}{$I L-6$} & F: ATGGTGTAAATCCCATGAAG \\
\hline & R: CCTCACGTCTTCTCCATCA \\
\hline \multirow[t]{2}{*}{$T N F-\alpha$} & F: CAGATGGGAAGGGAATGAAC \\
\hline & R: AGAGCATCAACGCAAAGGG \\
\hline \multirow[t]{2}{*}{ MyD 88} & F: CCGTCTTGTTGTCCAGTG \\
\hline & R: TGAGCAGAGGAACGAAGC \\
\hline \multirow[t]{2}{*}{ TRIF } & F: CCCATGTCTGTCTTGCTG \\
\hline & R: GTTGGTAGTCTGGCCTGA \\
\hline \multirow[t]{2}{*}{$N F-\kappa B$} & F: TCACGCAGACCTAAGACAT \\
\hline & R: GCAGATAGCAATTCAGATG \\
\hline \multirow[t]{2}{*}{ TLR1 } & F: GCCCTGACAGAGAATTGTT \\
\hline & R: TGATTTCATACGGCTGGTCA \\
\hline \multirow[t]{2}{*}{ TLR2 } & F: GGTGGCCAGAAAGCTACATC \\
\hline & R: GGGTGCAGATCAAGGACACT \\
\hline \multirow[t]{2}{*}{ TLR3 } & F: CTGCGGAATCTGACTGTCCT \\
\hline & R: TGCACATTTCCAAAGTCGAG \\
\hline \multirow[t]{2}{*}{ TLR4 } & F: GTTTGACATTGCTCGGTCCT \\
\hline & R: CCTCCTCAGATATCGGGACA \\
\hline \multirow[t]{2}{*}{ TLR5 } & F: TGCAACTTCATTTCCACTGC \\
\hline & R: GACACGAAGATTTGGCAGGT \\
\hline \multirow[t]{2}{*}{$T L R 7$} & F: TGCAATTGTGATGCAGTGTG \\
\hline & R: AACCAAGCTCCTCCCTTTGT \\
\hline \multirow[t]{2}{*}{ TLR15 } & F: CTACATCCGTAACCCGCATT \\
\hline & R: AGGGAGCAGTGCAGAAGTGT \\
\hline \multirow[t]{2}{*}{$T L R 21$} & F: TGCACTTGGCCAGAAACGAG \\
\hline & R: GGGTAGACAACCTGCACACG \\
\hline
\end{tabular}

Note: $F$, forward; $R$, reverse; $I F N-\gamma$, interferon- $\gamma ; I L-2$, interleukin- $2 ; I L$ 6, interleukin-6; $T N F-\alpha$, tumor necrosis factor- $\alpha$; TRIF, toll-interleukin-1 receptor domain-containing adapter-inducing interferon- $\beta ; M y D 88$, myeloid differential protein-88; $N F-\kappa B$, nuclear factor- $\kappa \mathrm{B} ; T L R$, Toll-like receptor

\section{Results}

\section{Se deficiency causes inflammatory injury in the broiler bursa of Fabricius}

In the present study, histopathological results showed that no pathological change in the bursa of Fabricius of the $\mathrm{C}$ group was observed (Fig. 1A). However, the whole tissue structure of the bursa of Fabricius was seriously destroyed and presented incomplete and disheveled in the L group. The lymph 
Fig. 1 Se deficiency causes inflammatory injury in the bursa of Fabricius. (A) The histopathology of the bursa of Fabricius in the $\mathrm{C}$ group. (B) The histopathology of the bursa of Fabricius in the L group and atrophied lymph nodes and epithelial mucosae (black arrows). Hemorrhage in the lymph nodes and mucosal lamina propria (yellow arrows). The enlarged interstitial space in the lymph nodes (red arrows). The results were similar in 3 biological replicates per group. $\mathrm{C}$, control. $\mathrm{L}$, low selenium

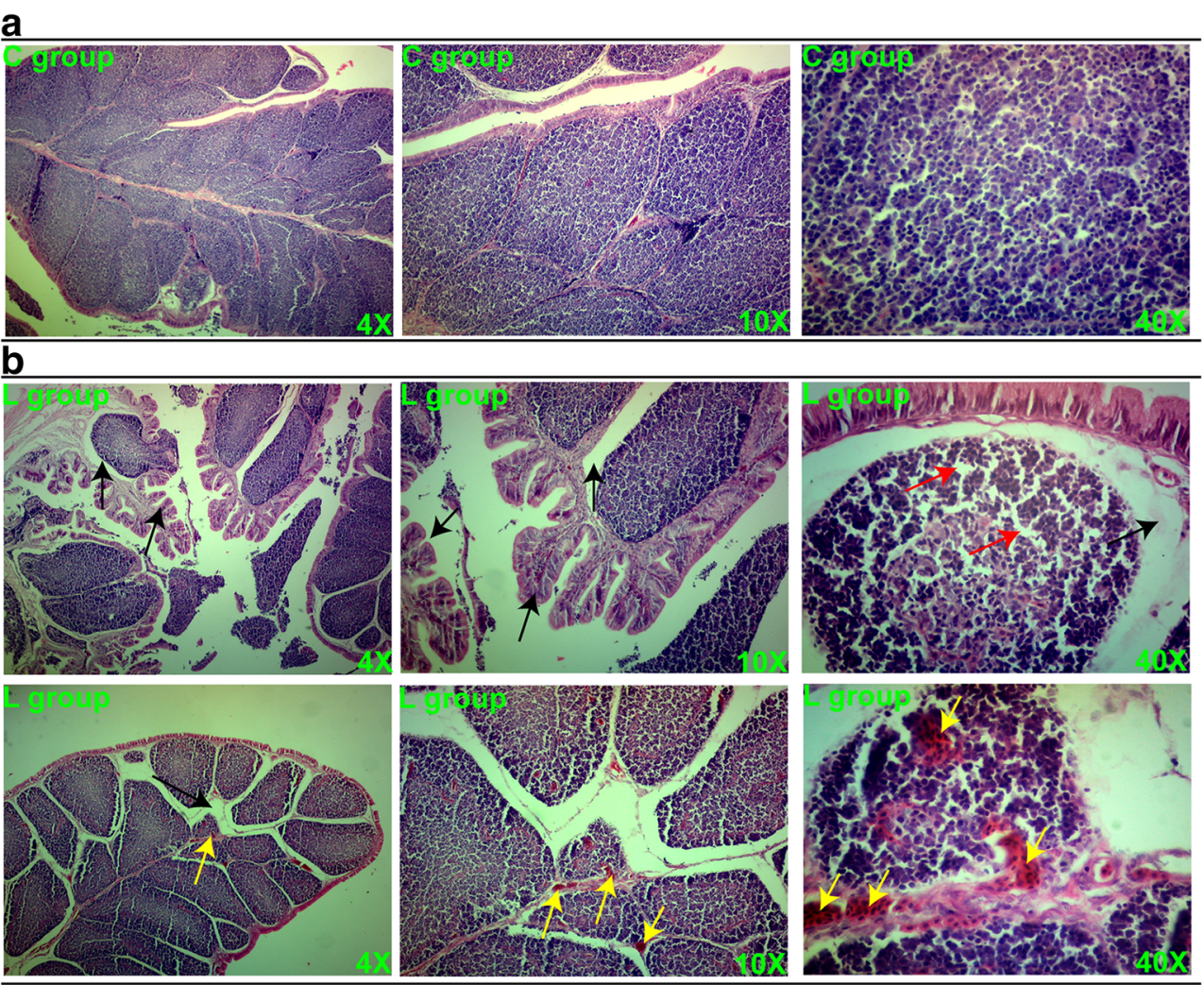

nodes were atrophied, and epithelial mucosae presented serrated atrophy in the L group. Multiple and severe hemorrhages obviously occurred in the lymph nodes and mucosal lamina propria of the L group. The interstitial space in the lymph nodes was enlarged in the L group, implying that edema occurred or the number of lymphocytes was reduced (Fig. 1B). The destroyed tissue structure, severe hemorrhage, and edema indicated that Se deficiency causes severe inflammatory injury in the broiler bursa of Fabricius.

\section{Se deficiency causes organelle damage in the broiler bursa of Fabricius}

In this study, the ultrastructural pathological results showed that no damaged organelles in the bursa of Fabricius of the $\mathrm{C}$ group were observed (Fig. 2A). However, in the L group, lymphocyte membranes were destroyed, the shaped nuclei were irregularly present, and nuclear membranes were damaged. Obviously, mitochondria were swelled, and some of their structures disappeared and were even vacuolized in the L group. Moreover, the endoplasmic reticulum was vacuolar and fractured. We also found that autophagic vacuoles might be produced in the L group (Fig. 2B). The foregoing results indicated that Se deficiency causes multiple organelle damage in the broiler bursa of Fabricius.

\section{Se deficiency markedly elevates proinflammatory cytokine mRNA expression in the broiler bursa of Fabricius}

In the present study, compared to the $\mathrm{C}$ group, IL- 6 mRNA expression was elevated in the $\mathrm{L}$ group and was especially markedly elevated on day $15(P<$ $0.0001)$ (Fig. 3A). Meanwhile, $I L-2$ mRNA expression was markedly elevated in the $\mathrm{L}$ group on day $15(P<$ 0.0001) (Fig. 3B). Additionally, $I F N-\gamma$ mRNA expression was increased in the $\mathrm{L}$ group and was especially markedly elevated on days 15 and $35(P<0.0001)$ (Fig. 3C). Compared to the $\mathrm{C}$ group, TNF- $\alpha$ mRNA expression was increased in the $\mathrm{L}$ group, with no difference (Fig. 3D). The foregoing data indicated that Se deficiency significantly initiates the mRNA expression of multiple proinflammatory cytokines in the broiler bursa of Fabricius, especially in the early stage of Se deficiency (on day 15).

\section{Se deficiency activates the TLR/MyD88/NF-KB signaling pathway in the broiler bursa of Fabricius}

Then, whether Se deficiency activated TLR signaling was determined. Compared to the C group, TLR-1 mRNA expression was elevated in the L group on days 15 and 35, and was especially markedly elevated on day 
Fig. 2 Se deficiency causes multiple organelle damage in the bursa of Fabricius. (A) The ultrastructural pathology of the bursa of Fabricius in the $\mathrm{C}$ group. (B) The ultrastructural pathology of the bursa of Fabricius in the L group. The destroyed membrane of the lymphocytes (purple arrows). The irregularly shaped nuclei (deep pink arrows).

Mitochondria were swollen, their structures disappeared and they showed vacuolization (green arrows). The vacuolar and fractured endoplasmic reticulum (blue arrows). The damaged nuclear membranes (red arrows) and autophagosomes (yellow arrow). The results were similar in 3 biological replicates per group. $\mathrm{C}$, control. L, low selenium

a

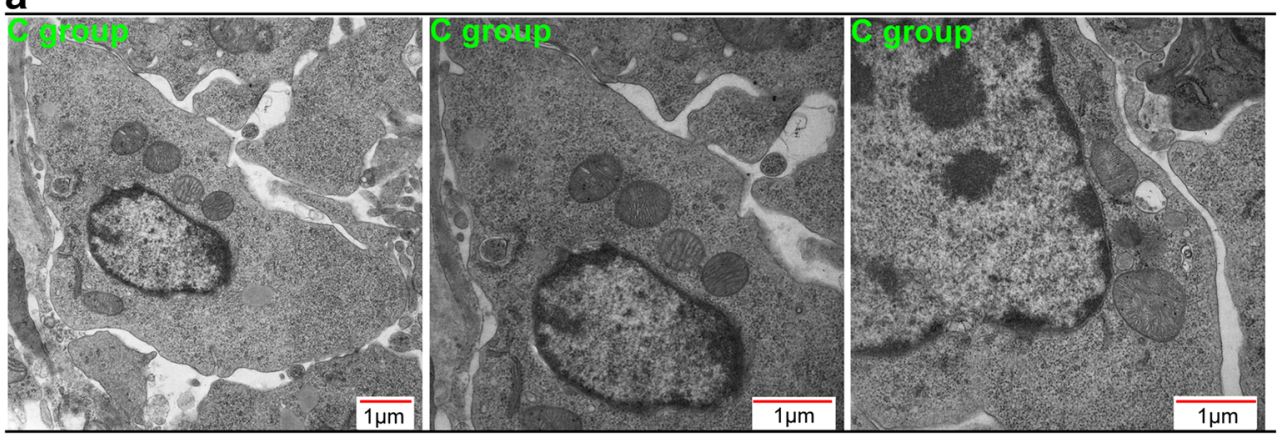

b
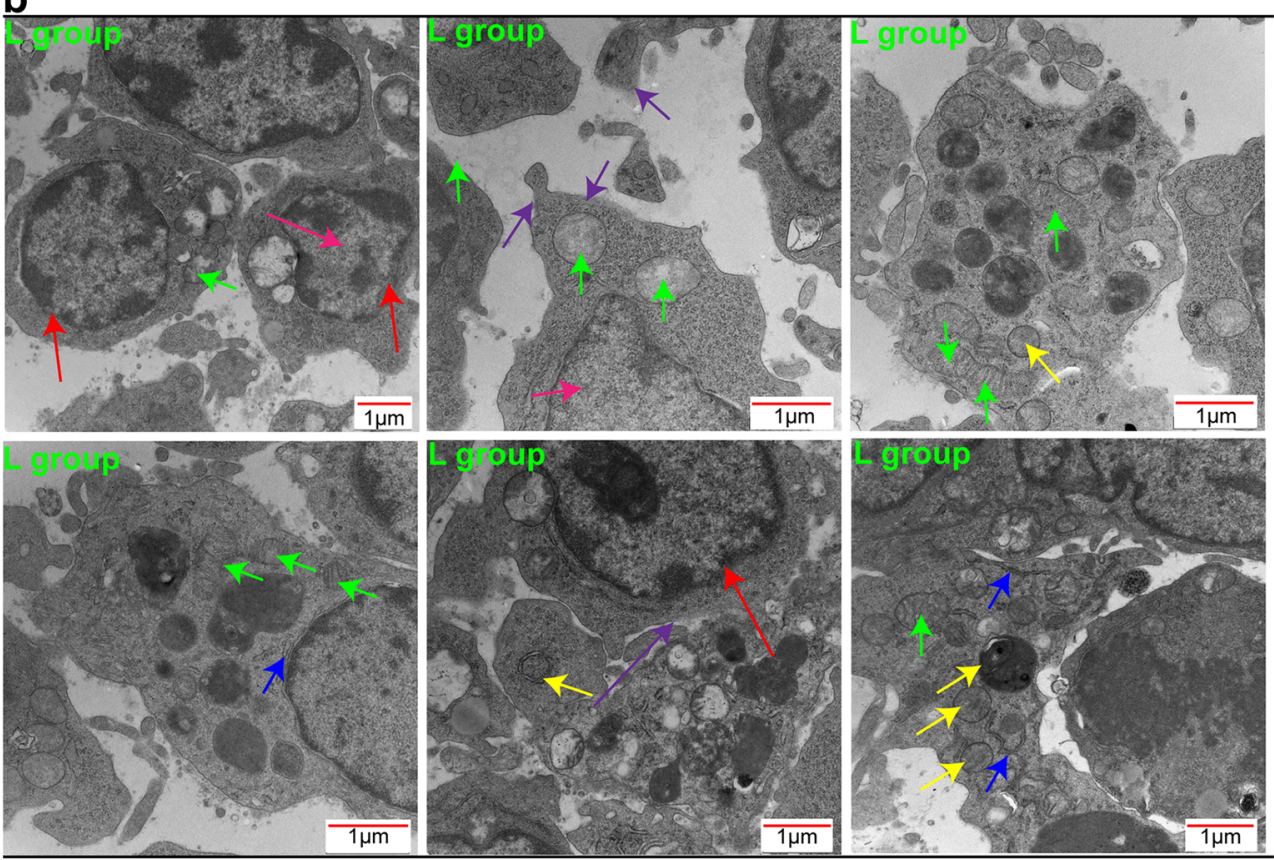

a

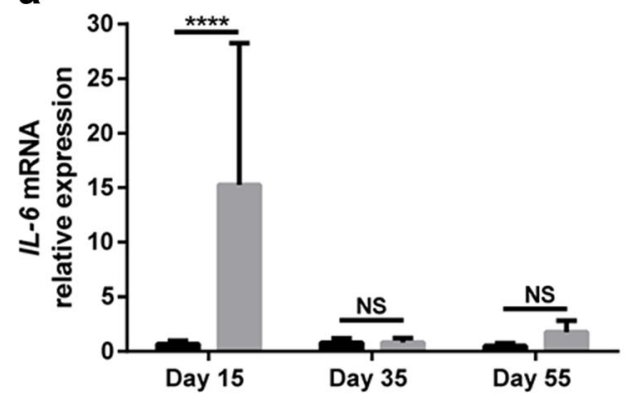

C group

Fig. 3 Se deficiency markedly elevates proinflammatory cytokine mRNA expression in the bursa of Fabricius. (A) The mRNA expression of $I L-6$ in the $\mathrm{C}$ and $\mathrm{L}$ groups $(n=5)$. (B) The mRNA expression of $I L-2$ in the $\mathrm{C}$ and L groups $(n=5)$. (C) The mRNA expression of $I F N-\gamma$ in the $\mathrm{C}$ and $\mathrm{L}$ groups $(n=5)$. (D) The mRNA expression of $T N F-\alpha$ in the $\mathrm{C}$ and $\mathrm{L}$ groups $(n=5)$. IL- 6 , interleukin-6. IL-2, interleukin-2. IFN- $\gamma$, interferon- $\gamma$. TNF- $\alpha$, tumor necrosis factor- $\alpha$. C, control. L, low selenium. $* * * *=$ $P<0.0001$. NS, nonsignificant

C

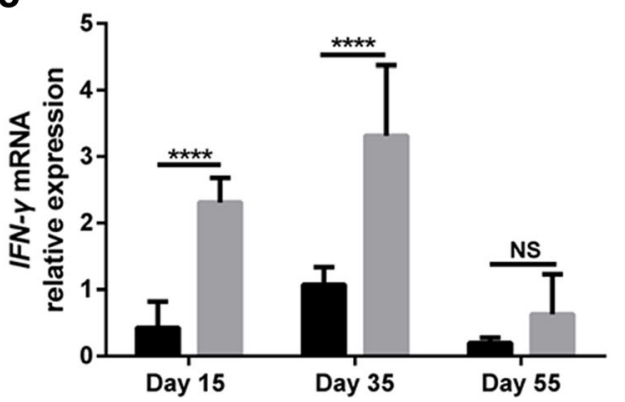

b

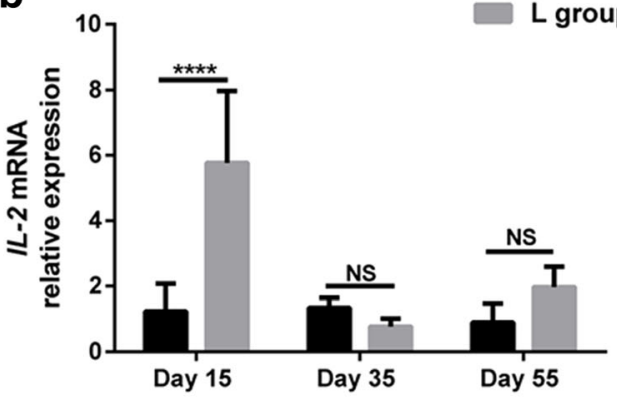

d

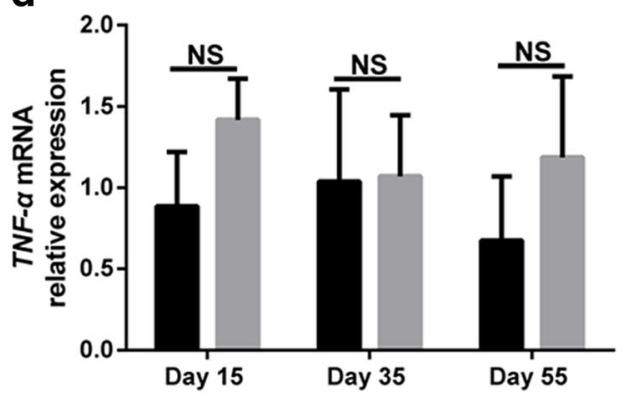


35 ( $P<0.05)$ (Fig. 4A); TLR-2 mRNA expression was elevated in the $\mathrm{L}$ group with no difference $(P>0.05)$ (Fig. 4B); both TLR-3 and TLR-4 mRNA expressions were markedly elevated in the $\mathrm{L}$ group on day $15(P$ $<0.0001$ and $P<0.01$ ) (Fig. 4C and D); TLR-5 mRNA expression was markedly elevated in the $\mathrm{L}$ group on day 55 ( $P<0.05)$ (Fig. 4E); TLR-7 mRNA expression was elevated in the $\mathrm{L}$ group on day 15 with no difference $(P>0.05)$, but markedly declined on day $35(P<$ 0.05 ) (Fig. 4F); TLR-15 mRNA expression was elevated in the $\mathrm{L}$ group from days 15 to 55 and was especially markedly elevated on day $15(P<0.001)$ (Fig. 4G), and TLR-21 mRNA expression was markedly elevated in the L group on day $35(P<0.01)$ (Fig. $4 \mathrm{H})$.
Based on the foregoing results of the activation of TLR signaling during the period of Se deficiency, we then determined whether the MyD88/NF-кB signaling pathway (the downstream signaling of TLR) was activated. $M y D 88$ mRNA expression was elevated in the $\mathrm{L}$ group compared to the $\mathrm{C}$ group and was especially markedly elevated on day 35 $(P<0.05)$ (Fig. 5A). TRIF mRNA expression was elevated in the $\mathrm{L}$ group compared to the $\mathrm{C}$ group and was especially markedly elevated on day $35(P<0.0001)$ (Fig. 5B). Compared to the $\mathrm{C}$ group, $N F-\kappa B$ mRNA expression was markedly elevated in the L group from days 15 to $55(P<$ $0.01, P<0.001$ ) (Fig. 5C). The foregoing data indicated that the TLR/MyD88/NF-KB signaling pathway was activated in the broiler bursa of Fabricius with Se deficiency.
Fig. 4 Se deficiency initiates TLR signaling in the bursa of Fabricius. (A) The mRNA expression of TLR- 1 in the $\mathrm{C}$ and L groups $(n=5)$. (B) The mRNA expression of TLR-2 in the $\mathrm{C}$ and L groups $(n=5)$. $(\mathbf{C})$ The mRNA expression of TLR-3 in the $\mathrm{C}$ and L groups $(n=5)$. (D) The mRNA expression of TLR-4 in the $\mathrm{C}$ and L groups $(n=5)$. (E) The mRNA expression of $T L R-5$ in the $\mathrm{C}$ and L groups $(n=5)$. (F) The mRNA expression of TLR-7 in the $\mathrm{C}$ and L groups $(n=5)$. $(\mathbf{G})$ The mRNA expression of TLR-15 in the $\mathrm{C}$ and L groups $(n=5)$. (H) The mRNA expression of TLR-21 in the C and L groups $(n=5)$. TLR, Toll-like receptor. C, control. L, low selenium. $*=P<0.05$. $* *=P<$ 0.01. $* * * *=P<0.0001$. NS, nonsignificant

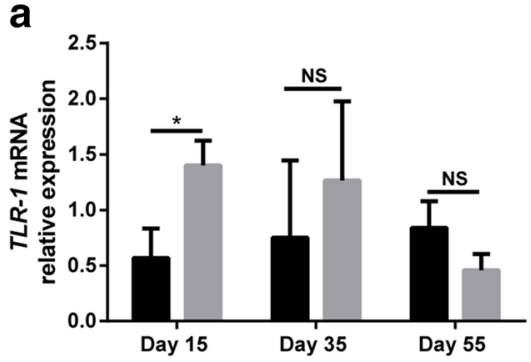

b
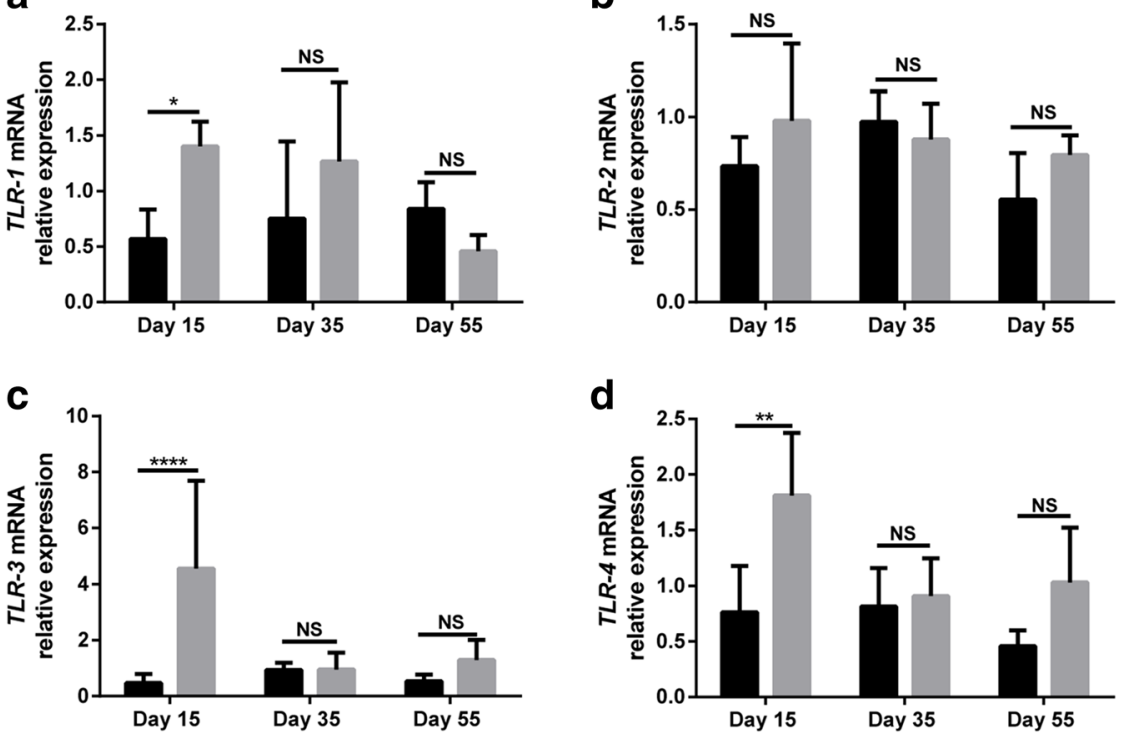

d

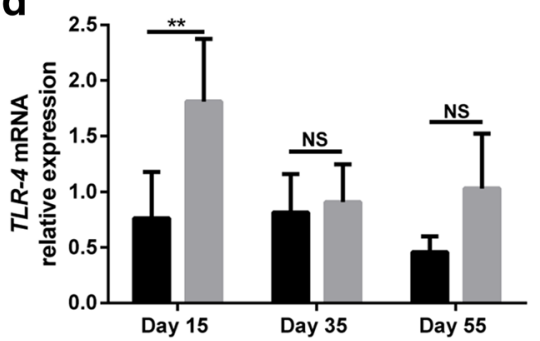

e

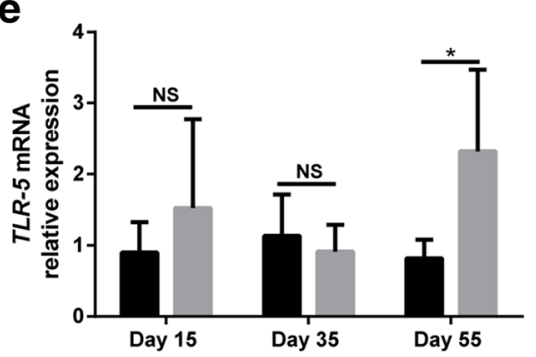

g

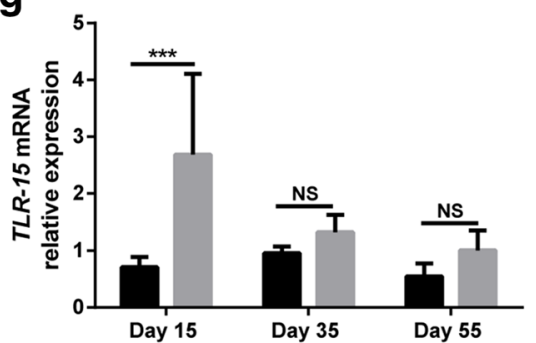

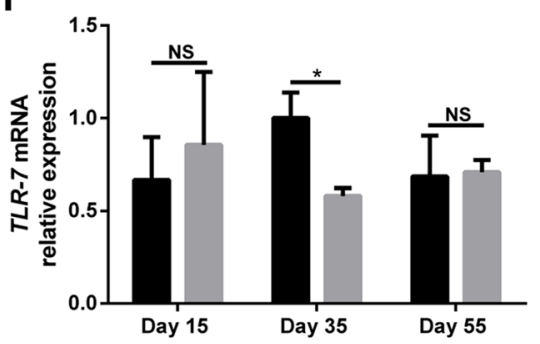

h

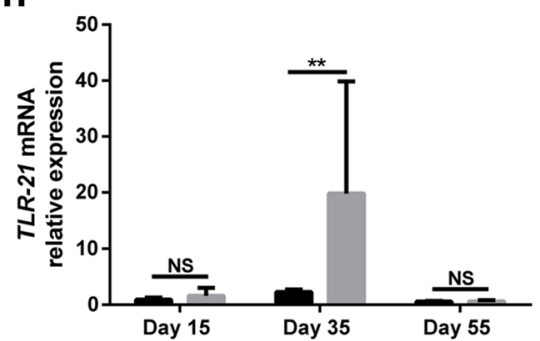

C group L group 
a

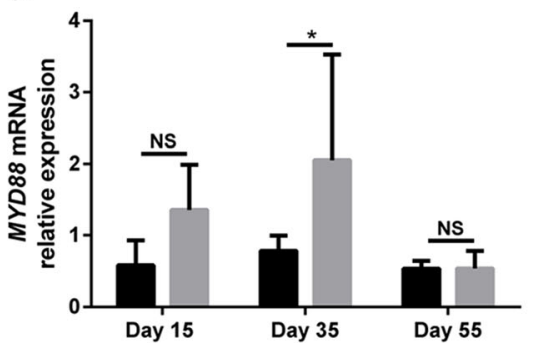

b

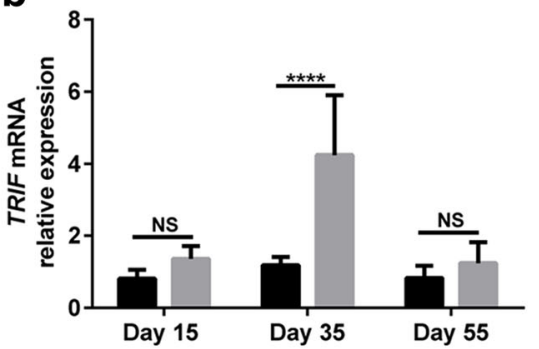

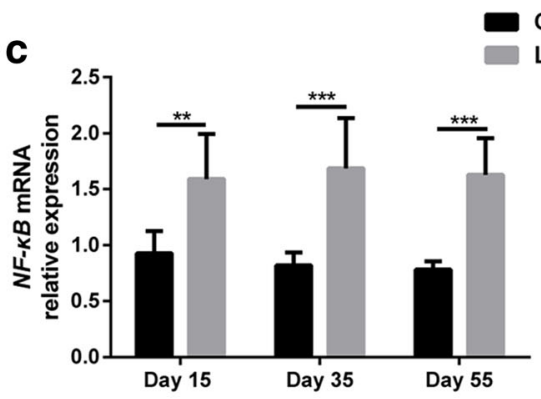

Fig. 5 Se deficiency activates MyD88/NF- $\mathrm{kB}$ signaling in the bursa of Fabricius. (A) The mRNA expression of $M y D 88$ in the $\mathrm{C}$ and $\mathrm{L}$ groups ( $n$ $=5$ ). (B) The mRNA expression of TRIF in the $\mathrm{C}$ and $\mathrm{L}$ groups $(n=5)$. (C) The mRNA expression of $N F-\kappa B$ in the $\mathrm{C}$ and $\mathrm{L}$ groups $(n=5)$. TRIF,

\section{Discussion}

Numerous studies have revealed that a diet with Se deficiency can cause inflammatory damage to broiler organs, such as the intestine, kidneys, and spleen $[6,10,17,19]$. In the present study, we revealed that severe inflammatory injury and multiple organelle damage obviously occurred in the broiler bursa of Fabricius of the L group via histopathology and ultrastructural pathology. The biological functions of the endoplasmic reticulum are the synthesis, modification and transportation of proteins, and the biosynthesis of lipids. The biological functions of the damaged endoplasmic reticulum might be intercepted in the broiler bursa of Fabricius in the later stage of Se deficiency. Mitochondria are the main sources of energy for aerobic respiration. Numerous studies found that the abnormal aerobic respiratory chain in mitochondria could lead to $\mathrm{O}_{2}{ }^{-}$accumulation, further resulting in an imbalance between oxidative and antioxidative responses [20,21]. In the present study, Se deficiency caused swelling in mitochondria, strongly suggesting that the aerobic respiratory chain in mitochondria might be destroyed and induce $\mathrm{O}_{2}^{-}$accumulation, which gives rise to excessive activation of the oxidative response. The imbalance between oxidative and antioxidative responses could induce inflammatory responses, which further contribute to organ damage.

Cytokines, which are divided into proinflammatory and anti-inflammatory cytokines based on their functions in the immune response, effectively regulate inflammatory response networks in humans and animals. IL- 6 and TNF- $\alpha$ are two kinds of critical proinflammatory cytokines in the early stage of innate immunity [15]. In the present study, we determined that Se deficiency increased $I L-6$ and $T N F-\alpha$ mRNA expression on day 15. IL-2 has been regarded as the key cytokine for the maturation of B lymphocytes abundantly existing in the bursa of Fabricius [14]. We also found that $I L-2$ mRNA expression was significantly increased in the $\mathrm{L}$ group on day 15 , which could also induce an inflammatory response in the bursa of Fabricius. IFN- $\gamma$ mediates macrophages to produce hydrogen peroxide and nitric oxide to prevent pathogens from toll-interleukin-1 receptor domain-containing adapter-inducing interferon- $\beta$. MyD88, myeloid differential protein- 88 . NF- $\mathrm{kB}$, nuclear factorkB. C, control. L, low selenium. $*=P<0.05$. $* *=P<0.01 . * * *=P$ $<0.001$. $* * * *=P<0.0001$. NS, nonsignificant

invading the body [22]. In this study, IFN- $\gamma$ mRNA expression was significantly increased in the $\mathrm{L}$ group on days 15 and 35 . The foregoing results revealed that enormous proinflammatory cytokine production was significantly increased during the early period of Se deficiency, which brings about excessive inflammation in the broiler bursa of Fabricius.

The result that enormous pro-inflammatory cytokine mRNA expression was obviously upregulated during the early process of Se deficiency prompted us to investigate whether Se deficiency induces the enormous pro-inflammatory cytokine production with high strength that is involved in the activation of the TLR/ MyD88/NF-KB signaling pathway. To prove this hypothesis, we measured the expression of 8 fundamental important $T L R$ mRNAs in the broiler bursa of Fabricius during the inflammatory response. We found that TLR-1,TLR-3,TLR-4, and TLR-15 mRNA expressions were markedly increased in the broiler bursa of Fabricius during the early period of Se deficiency. The results of this study were similar to those of our previous study in which Se deficiency caused excessive inflammation in the broiler spleen via the TLR-4/NF-kB signaling pathway [17]. Gao et al. found that Se deficiency facilitates inflammation following $S$. aureus infection by regulating TLR-2-related pathways in the mouse mammary gland, suggesting that TLR signaling also plays a vital role in the process of Se deficiency-induced inflammation [23]. The downstream TLR signaling pathway is the MyD88-mediated signaling pathway, containing MyD88 protein-mediated (classic MyD88) and TRIF protein-mediated (non-classic MyD88) signaling pathways [24]. In the present study, on day $15, M y D 88$ and TRIF mRNA expression was not different between the $\mathrm{C}$ and $\mathrm{L}$ groups. However, $M y D 88$ mRNA expression in the $\mathrm{L}$ group was much higher than that in the $\mathrm{C}$ group. The intragroup error in vivo is always larger than that in vitro. Thus, it is most likely that MyD88 signaling activated downstream signaling on day 15 . We uncovered that MyD88 and TRIF mRNA expressions were markedly increased in the $\mathrm{L}$ group. We also found that $N F-\kappa B$ mRNA expression was significantly increased in the broiler bursa of Fabricius with $\mathrm{Se}$ deficiency. The foregoing results obviously indicated that $\mathrm{Se}$ deficiency activates the TLR/MyD88/NF-KB signaling pathway 
in the broiler bursa of Fabricius. After much deliberation, we determined that Se deficiency induces an excessive inflammatory response that was dependent on TLRs.

Glutathione peroxidase (GPX) is an enzyme with antioxidant activity that neutralizes reactive oxygen species. GPX has been considered a Se-containing enzyme, and its activity is obviously decreased in many organs and tissues by feeding a Se-deficient diet, resulting in a lower antioxidant capacity [25-27]. The imbalance of oxidative and antioxidative responses could induce heat shock protein (HSP) production with high strength in animals. Emerging studies have indicated that endogenous HSP can also activate the TLR signaling pathway in humans and animals [28, 29]. For example, Hsp70 elicits rapid calcium flux, binds with high affinity to the plasma membrane, activates NF- $\mathrm{KB}$ and upregulates the expression of TNF- $\alpha$ in human monocytes [30]. Considering the published studies and findings in this study, we speculate that Se deficiency activates the TLR signaling pathway, and is probably due to the increased expression of HSPs in the bursa of Fabricius, which is our future subject as well.

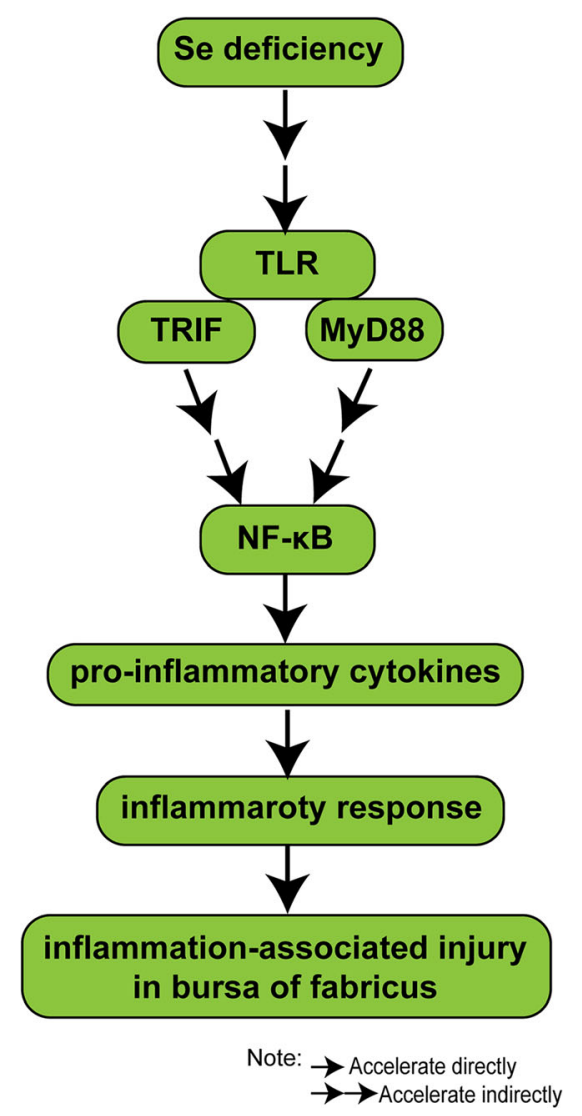

Fig. 6 A schematic diagram depicting the potential molecular mechanisms underlying Se deficiency-mediated inflammatory injury in the broiler bursa of Fabricius via activation of the TLR/MyD88/NF-KB signaling pathway. Se, selenium. TLR, Toll-like receptor. TRIF, tollinterleukin- 1 receptor domain-containing adapter-inducing interferon- $\beta$. MyD88, myeloid differential protein-88. NF- $\mathrm{kB}$, nuclear factor- $\mathrm{kB}$
It is believed that biological functions are strongly associated with organ and tissue structures and that they interact with each other. In the present study, Se deficiency initiated an excessive inflammatory response at 15 days, and persistent and excessive inflammation caused severe damage and destruction in the broiler bursa of Fabricius at 55 days. Because of the biological function impaired by damaged organ and tissue structures, proinflammatory cytokine expression was not significantly different at 55 days. We uncovered that Se deficiency obviously initiates the inflammatory response in the bursa of Fabricius, but the excessive inflammatory response causes severe injury to the broiler bursa of Fabricius, which decreases the immune response level in the later stage of Se deficiency. This is one of the reasons that $\mathrm{Se}$ deficiency impairs the maturation of the broiler bursa of Fabricius. This fact may be even more damaging for laying hens because of their longer growth period. Elucidation of the mechanism by which Se deficiency induced inflammatory injury in the broiler bursa of Fabricius from the perspective of initiating the TLR signaling pathway will help us to study further the underlying mechanism of Se deficiency-induced inflammatory injury in the bursa of Fabricius and to add Se to feed rationally during the process of livestock and poultry farming.

Indeed, there is a limitation in our present study. Given the considerable technical challenge of lacking antibodies, especially against chickens, we have not yet determined the protein level changes of proinflammatory cytokines and the TLR/MyD88/NF-KB signaling pathway in the broiler bursa of Fabricius. Additionally, this issue is a major dilemma in other studies on chickens, which urgently needs to be resolved in future studies.

Taken together, Se deficiency could markedly elevate enormous proinflammatory cytokine production by triggering the TLR/MyD88/NF-kB signaling pathway, which causes an excessive inflammatory response and thereby leads to severe inflammatory injury in the broiler bursa of Fabricius (Fig. 6).

Acknowledgements We thank the staff at the electron microscope laboratory of Harbin Veterinary Research Institute, Chinese Academy of Agricultural Sciences for the technical support they provided. We would also like to thank Professor Shimin Zheng for supplying histopathological technical support, which came from the laboratory of veterinary pathophysiology, College of Veterinary Medicine, Northeast Agricultural University.

Author contribution Yu Bai, Ruili Zhang and Qing Liu designed the research, performed most of the experiments, analyzed the data and drafted the manuscript. Rong Guo, Guangxing Li, Bin Sun, Di Zhang and Yang Chen helped perform the experiments. Xiaodan Huang modified the manuscript. All authors approved the final manuscript.

Funding This study was supported by the National Natural Science Foundation of China Youth Foundation (31602028) and the Postdoctoral Foundation of Heilongiiang Province (Project No. LBHQ19068). 


\section{Declarations}

Conflict of interest All authors declare no competing interests.

\section{References}

1. Sun W, Zhu J, Li S, Tang C, Zhao Q, Zhang J (2020) Selenium supplementation protects against oxidative stress-induced cardiomyocyte cell cycle arrest through activation of PI3K/AKT. Metallomics 1965-1979. https://doi.org/10.1039/d0mt00225a

2. Bakhshalinejad R, Hassanabadi A, Swick RA (2019) Dietary sources and levels of selenium supplements affect growth performance, carcass yield, meat quality and tissue selenium deposition in broilers. Anim Nutr 5(3):256-263. https://doi.org/10.1016/j.aninu. 2019.03.003

3. Sun X, S-z Y, Y-h Q, Z-j S, Wang C, H-f L (2020) Dietary supplementation with selenium-enriched earthworm powder improves antioxidative ability and immunity of laying hens. Poult Sci 99(11): 5344-5349. https://doi.org/10.1016/j.psj.2020.07.030

4. He S, Yu Q, He Y, Hu R, Xia S, He J (2019) Dietary resveratrol supplementation inhibits heat stress-induced high-activated innate immunity and inflammatory response in spleen of yellow-feather broilers. Poult Sci 98(12):6378-6387. https://doi.org/10.3382/ps/ pez471

5. Fan R-F, Liu J-X, Yan Y-X, Wang L, Wang Z-Y (2020) Selenium relieves oxidative stress, inflammation, and apoptosis within spleen of chicken exposed to mercuric chloride. Poult Sci 99(11):5430 5439. https://doi.org/10.1016/j.psj.2020.08.031

6. Wan N, Xu Z, Chi Q, Hu X, Pan T, Liu T, Li S (2019) MicroRNA33-3p involved in selenium deficiency-induced apoptosis via targeting ADAM10 in the chicken kidney. J Cell Physiol 234(8): 13693-13704. https://doi.org/10.1002/jcp.28050

7. Bi C-L, Zhang S-J, Shen Y-Z, Pauline M, Li H, Tang H (2020) Selenium plays an anti-inflammatory role by regulation NLRP3 inflammasome in Staphylococcus aureus-infected mouse mammary gland. Biol Trace Elem Res 199(2):604-610. https://doi.org/10. 1007/s12011-020-02166-Z

8. Qing Z, Kaixin Z, Yanfei H, Yiming Z, Hua X, Ling Z, Guangliang S, Shu L (2020) MicroRNA-223 triggers inflammation in porcine aorta by activating NLRP3 inflammasome under selenium deficiency. J Cell Physiol 236:4555-4564. https://doi.org/10.1002/jcp. 30178

9. Zheng S, Zhao J, Xing H, Xu S (2019) Oxidative stress, inflammation, and glycometabolism disorder-induced erythrocyte hemolysis in selenium-deficient exudative diathesis broilers. J Cell Physiol 234(9):16328-16337. https://doi.org/10.1002/jcp.28298

10. Wu C, Xu Z, Huang K (2016) Effects of dietary selenium on inflammation and hydrogen sulfide in the gastrointestinal tract in chickens. Biol Trace Elem Res 174(2):428-435. https://doi.org/ 10.1007/s12011-016-0735-y

11. Shojadoost B, Kulkarni RR, Yitbarek A, Laursen A, TahaAbdelaziz K, Negash Alkie T, Barjesteh N, Quinteiro-Filho WM, Smith TK, Sharif S (2019) Dietary selenium supplementation enhances antiviral immunity in chickens challenged with low pathogenic avian influenza virus subtype H9N2. Vet Immunol Immunopathol 207:62-68. https://doi.org/10.1016/j.vetimm.2018. 12.002

12. Alexander J, Tinkov A, Strand TA, Alehagen U, Skalny A, Aaseth J (2020) Early nutritional interventions with zinc, selenium and vitamin $\mathrm{D}$ for raising anti-viral resistance against progressive COVID-19. Nutrients 12(8):2358-2370. https://doi.org/10.3390/ nu12082358
13. Jia X, Liu B, Bao L, Lv Q, Li F, Li H, An Y, Zhang X, Cao B, Wang C (2018) Delayed oseltamivir plus sirolimus treatment attenuates $\mathrm{H} 1 \mathrm{~N} 1$ virus-induced severe lung injury correlated with repressed NLRP3 inflammasome activation and inflammatory cell infiltration. PLoS Pathog 14(11):e1007428. https://doi.org/10. 1371/journal.ppat.1007428

14. Iwasaki A, Pillai PS (2014) Innate immunity to influenza virus infection. Nat Rev Immunol 14(5):315-328. https://doi.org/10. 1038/nri3665

15. Bi Y, Tan S, Yang Y, Wong G, Zhao M, Zhang Q, Wang Q, Zhao X, Li L, Yuan J, Li H, Li H, Xu W, Shi W, Quan C, Zou R, Li J, Zheng H, Yang L, Liu WJ, Liu D, Wang H, Qin Y, Liu L, Jiang C, Liu W, Lu L, Gao GF, Liu Y (2018) Clinical and immunological characteristics of human infections with H5N6 avian influenza virus. Clin Infect Dis 68:1100-1109. https://doi.org/10.1093/cid/ ciy681

16. Zhang Y, Zhang J, Bao J, Tang C, Zhang Z (2020) Selenium deficiency induced necroptosis, Th1/Th2 imbalance, and inflammatory responses in swine ileum. J Cell Physiol 236(1):222-234. https:// doi.org/10.1002/jcp.29836

17. Zhang R, Guo R, Liu Q, Li G, Sun B, Huang X (2020) Selenium deficiency via the TLR4/TRIF/NF- $\mathrm{BB}$ signaling pathway leading to inflammatory injury in chicken spleen. Biol Trace Elem Res 199: 693-702. https://doi.org/10.1007/s12011-020-02173-0

18. Huang X, Chang Y, Sun B, Zhang J, Gao Y, Li G (2017) Selenium deficiency induced injury in chicken muscular stomach by downregulating selenoproteins.pdf. Biol Trace Elem Res. https://doi.org/ 10.1007/s12011-017-0946-X

19. Zhang J-1 XB, X-d H, Gao Y-h, Chen Y, A-s S (2015) Selenium deficiency affects the mRNA expression of inflammatory factors and selenoprotein genes in the kidneys of broiler chicks. Biol Trace Elem Res 171(1):201-207. https://doi.org/10.1007/s12011-0150512-3

20. Demirci K, Nazıroğlu M, Övey İS, Balaban H (2016) Selenium attenuates apoptosis, inflammation and oxidative stress in the blood and brain of aged rats with scopolamine-induced dementia. Metab Brain Dis 32(2):321-329. https://doi.org/10.1007/s11011-0169903-1

21. Gao X-j, Tang B, Liang H-h, Yi L, Z-g W (2019) Selenium deficiency inhibits micRNA-146a to promote ROS-induced inflammation via regulation of the MAPK pathway in the head kidney of carp. Fish Shellfish Immunol 91:284-292. https://doi.org/10.1016/ j.fsi.2019.05.039

22. Wang W, Yang P, Zhong Y, Zhao Z, Xing L, Zhao Y, Zou Z, Zhang Y, Li C, Li T, Wang C, Wang Z, Yu X, Cao B, Gao X, Penninger JM, Wang X, Jiang C (2013) Monoclonal antibody against CXCL-10/IP-10 ameliorates influenza A (H1N1) virus induced acute lung injury. Cell Res 23(4):577-580. https://doi.org/ $10.1038 / \mathrm{cr} .2013 .25$

23. Gao X, Zhang Z, Li Y, Shen P, Hu X, Cao Y, Zhang N (2016) Selenium deficiency facilitates inflammation following $S$. aureus infection by regulating TLR2-related pathways in the mouse mammary gland. Biol Trace Elem Res 172(2):449-457. https://doi.org/ 10.1007/s12011-015-0614-y

24. Bi C-L, Wang H, Wang Y-J, Sun J, Dong J-S, Meng X, Li J-J (2016) Selenium inhibits Staphylococcus aureus-induced inflammation by suppressing the activation of the NF-KB and MAPK signalling pathways in RAW264.7 macrophages. Eur J Pharmacol 780:159-165. https://doi.org/10.1016/j.ejphar.2016.03.044

25. McGarry T, Biniecka M, Veale DJ, Fearon U (2018) Hypoxia, oxidative stress and inflammation. Free Radic Biol Med 125:1524. https://doi.org/10.1016/j.freeradbiomed.2018.03.042

26. Gao J, Tian X, Yan X, Wang Y, Wei J, Wang X, Yan X, Song G (2020) Selenium exerts protective effects against fluoride-induced apoptosis and oxidative stress and altered the expression of Bcl-2/ 
caspase family. Biol Trace Elem Res 199(2):682-692. https://doi. org/10.1007/s12011-020-02185-w

27. Jin C, Cleveland JC, Ao L, Li J, Zeng Q, Fullerton DA, Meng X (2014) Human myocardium releases heat shock protein 27 (HSP27) after global ischemia: the proinflammatory effect of extracellular HSP27 through Toll-like Receptor (TLR)-2 and TLR4. Mol Med 20(1):280-289. https://doi.org/10.2119/molmed.2014.00058

28. Gao X-j, Tang B, Liang H-h, Yi L, Z-g W (2019) Selenium deficiency induced an inflammatory response by the HSP60 - TLR2MAPKs signalling pathway in the liver of carp. Fish Shellfish Immunol 87:688-694. https://doi.org/10.1016/j.fsi.2019.02.017
29. Terai K, Mochizuki N (2011) Heat shock proteins regulates cardiomyocytes via Toll-like receptors. Circ J 75(10):2322-2323. https://doi.org/10.1253/circj.CJ-11-0647

30. Yuan X, Zhu J, Kang Q, He X, Guo D (2019) Protective effect of hesperidin against sepsis-induced lung injury by inducing the heatstable protein 70 (Hsp70)/Toll-Like Receptor 4 (TLR4)/Myeloid Differentiation Primary Response 88 (MyD88) pathway. Med Sci Monit 25:107-114. https://doi.org/10.12659/msm.912490

Publisher's Note Springer Nature remains neutral with regard to jurisdictional claims in published maps and institutional affiliations. 